\title{
HERSCHEL OBSERVATIONS OF THE T CHA TRANSITION DISK: CONSTRAINING THE OUTER DISK PROPERTIES
}

\author{
Lucas A. Cieza ${ }^{1,7}$, Johan Olofsson ${ }^{2}$, Paul M. Harvey ${ }^{3}$, Christophe Pinte ${ }^{4}$, Bruno Merín ${ }^{5}$, Jean-Charles Augereau ${ }^{4}$, \\ Neal J. Evans II $^{3}$, Joan Najita ${ }^{6}$, Thomas Henning ${ }^{2}$, and Francois Ménard ${ }^{4}$ \\ ${ }^{1}$ Institute for Astronomy, University of Hawaii at Manoa, Honolulu, HI 96822, USA; lcieza@ ifa.hawaii.edu \\ ${ }^{2}$ Max Planck Institut für Astronomie, Königstuhl 17, 69117 Heidelberg, Germany \\ ${ }^{3}$ Department of Astronomy, University of Texas at Austin, Austin, TX 78712, USA \\ ${ }^{4}$ UJF-Grenoble 1/CNRS-INSU, Institut de Planétologie et d'Astrophysique de Grenoble (IPAG) UMR 5274, Grenoble, F-38041, France \\ ${ }^{5}$ Herschel Science Centre, European Space Agency (ESAC), P.O. Box 78, 28691 Villanueva de la Cañada, Madrid, Spain \\ ${ }^{6}$ National Optical Astronomy Observatory, 950 N. Cherry Avenue, Tucson, AZ 86719, USA \\ Received 2011 August 26; accepted 2011 September 26; published 2011 October 18
}

\begin{abstract}
T Cha is a nearby $(d \sim 100 \mathrm{pc})$ transition disk known to have an optically thin gap separating optically thick inner and outer disk components. Huélamo et al. recently reported the presence of a low-mass object candidate within the gap of the $\mathrm{T}$ Cha disk, giving credence to the suspected planetary origin of this gap. Here we present the Herschel photometry $(70,160,250,350$, and $500 \mu \mathrm{m})$ of T Cha from the "Dust, Ice, and Gas in Time" Key Program, which bridges the wavelength range between existing Spitzer and millimeter data and provide important constraints on the outer disk properties of this extraordinary system. We model the entire optical to millimeter wavelength spectral energy distribution (SED) of T Cha (19 data points between 0.36 and $3300 \mu \mathrm{m}$ without any major gaps in wavelength coverage). T Cha shows a steep spectral slope in the far-IR, which we find clearly favors models with outer disks containing little or no dust beyond $\sim 40 \mathrm{AU}$. The full SED can be modeled equally well with either an outer disk that is very compact (only a few AU wide) or a much larger one that has a very steep surface density profile. That is, T Cha's outer disk seems to be either very small or very tenuous. Both scenarios suggest a highly unusual outer disk and have important but different implications for the nature of T Cha. Spatially resolved images are needed to distinguish between the two scenarios.
\end{abstract}

Key words: circumstellar matter - planet-disk interactions - protoplanetary disks - stars: individual (T Cha) submillimeter: planetary systems

Online-only material: color figures

\section{INTRODUCTION}

Transition disks were first identified by the Infrared Astronomical Satellite as objects with little or no excess emission at $\lambda<10 \mu \mathrm{m}$ and a significant excess at $\lambda \geqslant 10 \mu \mathrm{m}$ (Strom et al. 1989). This broad definition encompasses a wide range of spectral energy distribution (SED) morphologies (Najita et al. 2007). T Cha belongs to a subclass of transition disks known as cold disks, which are characterized by a steep rise in the SED between $\sim 10$ and $\sim 30 \mu \mathrm{m}$ (Brown et al. 2007; Merín et al. 2010). It is now well established, from both SED modeling and direct (sub)millimeter imaging, that the peculiar SED shapes of cold disks are due to the presence of large inner holes or gaps in their dusty disks. Inner holes can be produced by a number of processes, including grain growth, photoevaporation, and dynamical interactions with (sub)stellar or planetary mass companions (see Williams \& Cieza 2011 for a recent review). However, objects such as T Cha, LkCa 15, and UX Tau are believed to have an optically thick inner disk within a much larger inner hole (Olofsson et al. 2011; Espaillat et al. 2010). Optically thin gaps separating optically thick inner and outer disk components are difficult to explain by anything other than the dynamical interaction of substellar or planetary mass objects embedded within the disk. The discovery of what seems to be either a young planet or a brown dwarf surrounded by a significant amount of dust within the gap of the T Cha disk (Huélamo et al. 2011) supports the suspected planetary origin of

\footnotetext{
7 Sagan Fellow.
}

the gap. Detailed studies of the properties of the T Cha disk thus become highly desirable to further our understanding of protoplanetary disk evolution and planet formation. Using Spitzer photometry and spectroscopy, Brown et al. (2007) first modeled $\mathrm{T}$ Cha with an inner disk extending from 0.08 to $0.2 \mathrm{AU}$ and an outer disk extending from 15 to $300 \mathrm{AU}$. More recently, Olofsson et al. (2011) simultaneously modeled the SED of T Cha and near-IR interferometric observations obtained with the AMBER instrument at the Very Large Telescope Interferometer (Scholler 2007). They spatially resolved the narrow inner dusty disk and constrained its inner edge to be $\sim 0.1 \mathrm{AU}$ from the star.

Here we present the Herschel photometry (at 70, 160, 250, 350 , and $500 \mu \mathrm{m}$ ) of $\mathrm{T}$ Cha and model its entire optical to millimeter wavelength SED. We focus our analysis on the properties of the outer disk (i.e., beyond the gap), which is the region that is both the best probed by Herschel data and the least constrained by previous studies.

\section{OBSERVATIONS}

\subsection{Herschel Observations and Data Reduction}

We obtained far-IR and submillimeter wavelength photometry for T Cha as part of the Dust, Ice, and Gas in Time (DIGIT) Open Time Herschel Key Program using both the Photodetector Array Camera and Spectrometer (PACS; 70 and $160 \mu \mathrm{m}$; Poglitsch et al. 2010) and the Spectral and Photometric Imaging Receiver (SPIRE; 250, 350, and $500 \mu \mathrm{m}$; Griffin et al. 2010). 
Table 1

T Cha Photometry Data

\begin{tabular}{|c|c|c|c|c|}
\hline $\begin{array}{l}\text { Wavelength } \\
(\mu \mathrm{m})\end{array}$ & $\begin{array}{l}\text { Flux } \\
(\mathrm{mJy})\end{array}$ & $\begin{array}{l}\text { Error } \\
(\mathrm{mJy})\end{array}$ & Telescope & Reference \\
\hline 0.36 & $6.80 \mathrm{e}+00$ & $10 \%$ & ESO $50 \mathrm{~cm}$ & 1 \\
\hline 0.44 & $4.03 e+01$ & $10 \%$ & ESO $50 \mathrm{~cm}$ & 1 \\
\hline 0.55 & $1.12 \mathrm{e}+02$ & $10 \%$ & ESO $50 \mathrm{~cm}$ & 1 \\
\hline 0.64 & $2.01 \mathrm{e}+02$ & $10 \%$ & ESO $50 \mathrm{~cm}$ & 1 \\
\hline 0.79 & $3.61 e+02$ & $10 \%$ & ESO $50 \mathrm{~cm}$ & 1 \\
\hline 1.2 & $4.20 \mathrm{e}+02$ & $10 \%$ & 2MASS & 2 \\
\hline 1.6 & $8.05 e+02$ & $10 \%$ & 2MASS & 2 \\
\hline 2.2 & $1.10 \mathrm{e}+03$ & $10 \%$ & 2MASS & 2 \\
\hline 3.6 & $1.49 \mathrm{e}+03$ & $10 \%$ & Spitzer & 3 \\
\hline 4.5 & $1.32 \mathrm{e}+03$ & $10 \%$ & Spitzer & 3 \\
\hline 5.8 & $1.07 \mathrm{e}+\mathrm{e} 3$ & $10 \%$ & Spitzer & 3 \\
\hline 8.0 & $6.66 e+02$ & $10 \%$ & Spitzer & 3 \\
\hline 70 & $5.06 e+03$ & $15 \%$ & Herschel & 4 \\
\hline 160 & $2.97 e+03$ & $15 \%$ & Herschel & 4 \\
\hline 250 & $1.78 \mathrm{e}+03$ & $20 \%$ & Herschel & 4 \\
\hline 350 & $1.06 e+03$ & $20 \%$ & Herschel & 4 \\
\hline 500 & $6.60 \mathrm{e}+02$ & $20 \%$ & Herschel & 4 \\
\hline 1300 & $1.05 \mathrm{e}+02$ & $15 \%$ & SEST & 5 \\
\hline 3300 & $6.40 \mathrm{e}+00$ & $15 \%$ & ATCA & 5 \\
\hline
\end{tabular}

References. (1) Alcalá et al. 1993; (2) Skrutskie et al. 2006; (3) Brown et al. 2007; (4) this work; (5) Lommen et al. 2007.

The PACS observations (OBS ID $=1342209063$ ) were obtained on 2010 November 5, using the Mini Scan Map mode, a repetition factor of three, and a scan angle of $70^{\circ}$. Similarly, the SPIRE observations (OBS ID = 1342203636) were taken on 2010 August 24, using the Small Scan Map mode, and a repetition factor of three. Both the PACS and SPIRE data were processed using Herschel Interactive Processing Environment (Ott 2010) version 7.1. The PACS beam sizes at 70 and $160 \mu \mathrm{m}$ are 5.5 and $11^{\prime \prime}$, and the data were resampled to $1^{\prime \prime}$ and $2^{\prime \prime}$ pixel images, respectively. SPIRE beam sizes at 250,350 , and $500 \mu \mathrm{m}$ are $18^{\prime \prime} .1$, $25^{\prime \prime} .2$, and $36^{\prime \prime} .6$, respectively, and the images were resampled to $6^{\prime \prime}, 10^{\prime \prime}$, and $14^{\prime \prime}$ pixel images, respectively.

T Cha was detected with good signal to noise $(\gtrsim 15-50)$ at all PACS and SPIRE wavelengths. PACS and SPIRE fluxes were derived with a psf-fitting routine essentially identical to the c2dphot software used for the c2d Spitzer Legacy Program ${ }^{8}$ (Evans et al. 2007). We used the empirical psf's posted by the NASA Herschel Science Center ${ }^{9}$ and found that they gave a good match to the actual images of $\mathrm{T}$ Cha. In addition, we checked that aperture photometry gave similar results and assigned the final uncertainties based on the $10 \%$ and $15 \%$ error estimated for PACS and SPIRE absolute photometry, respectively. We also added an additional 5\% uncertainty to reflect incomplete knowledge of the psf and the agreement between the psf-fitting and the aperture photometry. The resulting Herschel photometry for T Cha is listed in Table 1.

\subsection{Photometry Data from the Literature}

In order to construct the full SED of T Cha, we collected optical, near-IR, mid-IR, and millimeter wavelength photometry from the literature, which are also listed in Table 1. The full optical to millimeter wavelength SED for T Cha is shown in Figure 1. There are now a total of 19 photometry points without

\footnotetext{
8 Available at the Infra-Red Science Archive, http://irsa.ipac.caltech.edu/data/SPITZER/C2D/doc/c2d_del_document.pdf 9 https://nhscsci.ipac.caltech.edu/sc/index.php/
}
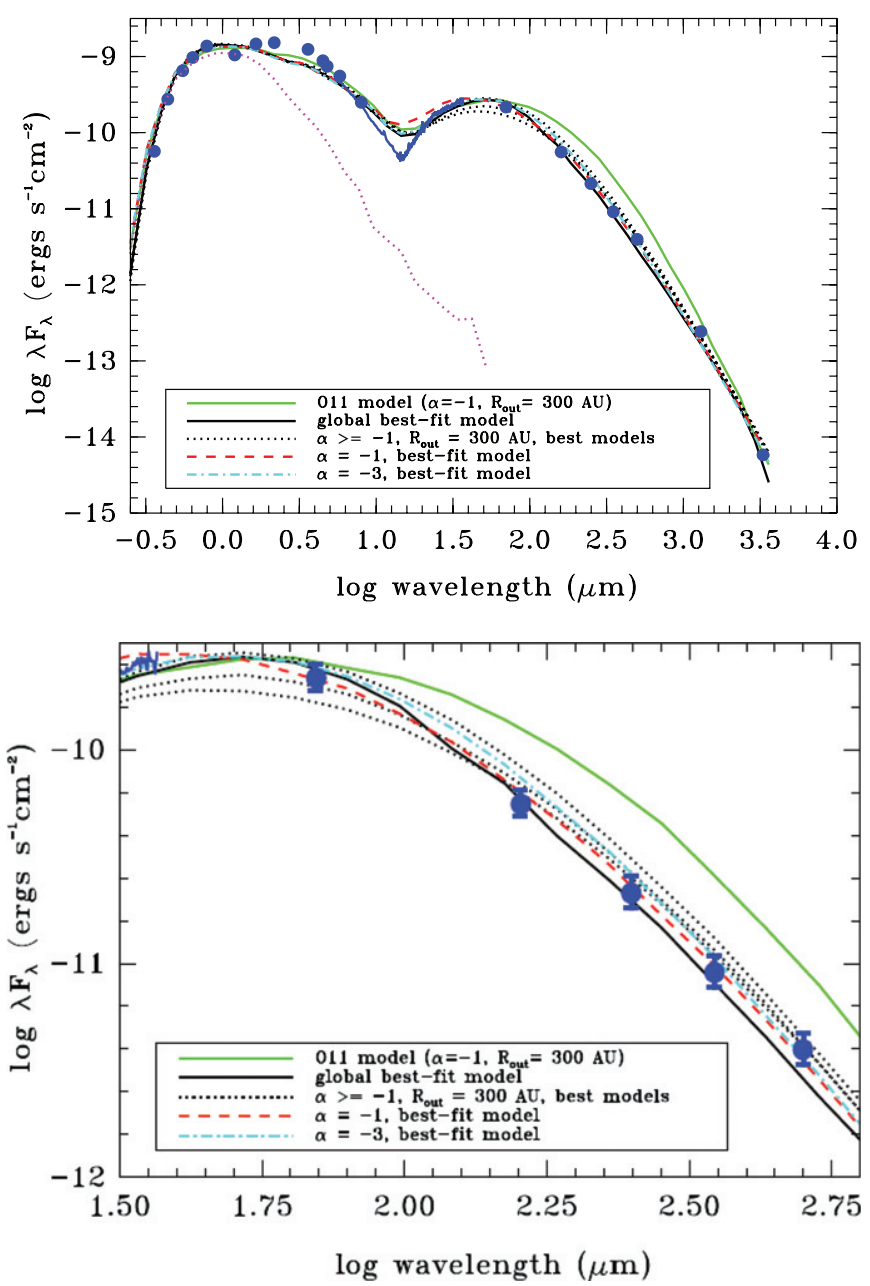

Figure 1. Top panel: the full spectral energy distribution (SED) of T Cha and a variety of models as labeled. The $\mathrm{O} 11$ model fits all previously available data but greatly overestimates the new 160, 250, 350, and $500 \mu \mathrm{m}$ fluxes, illustrating the power of Herschel to completely rule out certain models. Lower panel: the SED region sampled by Herschel. While the global bestfit model has a far-IR slope slightly steeper than observed, all disk models with a shallow surface density profile and a large outer radius, including the O11 model, result in far-IR colors that are much too red. The surface density profile of the outer disk and its inner and outer radii are degenerate parameters. Equally good fits can be obtained by either adopting a small outer radius $\left(R_{\text {out }}=30 \mathrm{AU}\right.$, red dashed line) or a very steep surface density profile ( $\alpha=-3$, light blue dot-dashed line).

(A color version of this figure is available in the online journal.)

major gaps in wavelength coverage between 0.36 and $3300 \mu \mathrm{m}$. In addition to the above photometry, the T Cha SED is well sampled by a Spitzer Infrared Spectrograph (IRS) spectrum, covering the 5.2 to $38 \mu \mathrm{m}$ region (Brown et al. 2007). The sharp dip in the SED around $15 \mu \mathrm{m}$ indicates the presence of a wide gap in the disk.

\section{DISK MODEL}

The starting point of our study is the model presented by Olofsson et al. (2011, O11 hereafter). Following O11, we use the Monte Carlo radiative transfer code MCFOST (Pinte et al. 2006, 2009). We parameterize the structure of the T Cha disk with the following parameters: the inner and outer radii $\left(R_{\text {in }}\right.$ and $R_{\text {out }}$, respectively), the index $\alpha$ for the surface density profile $\left(\Sigma(r)=\Sigma\left(r / r_{0}\right)^{\alpha}\right)$ and a disk scale height $H(r)$ assuming a vertical Gaussian distribution $\left(\exp \left[-z^{2} / 2 H(r)^{2}\right]\right)$, appropriate for a disk in hydrostatic equilibrium. The disk's flaring is 
Table 2

Fixed Parameters from 011

\begin{tabular}{lc}
\hline \hline Parameter & Adopted value \\
\hline Stellar $T_{\text {eff }}(\mathrm{K})$ & 5400 \\
Stellar radius $\left(R_{\odot}\right)$ & 1.3 \\
Stellar mass $\left(M_{\odot}\right)$ & 1.5 \\
Distance $(\mathrm{pc})$ & 100 \\
Inclination $($ deg $)$ & 60 \\
Grain-size distribution slope, $p$ & -3.5 \\
Inner disk $R_{\text {in }}(\mathrm{AU})$ & 0.13 \\
Inner disk $R_{\text {out }}(\mathrm{AU})$ & 0.17 \\
Inner disk $M_{\text {dust }}\left(M_{\odot}\right)$ & $10^{-9}$ \\
Inner disk $a_{\min }(\mu \mathrm{m})$ & 0.1 \\
Inner disk $a_{\max }(\mu \mathrm{m})$ & 10 \\
Outer disk $a_{\min }(\mu \mathrm{m})$ & 0.01 \\
\hline
\end{tabular}

described by a power law determining the scale height as a function of the radius $\left(H(r)=H_{0}\left(r / r_{0}\right)^{\beta}\right)$. The dust content is described by a differential power law for the grain-size distribution $\left(\mathrm{d} n(\mathrm{a}) \propto \mathrm{a}^{p} \mathrm{~d} a\right)$, between the minimum $\left(a_{\min }\right)$ and maximum $\left(a_{\max }\right)$ grain sizes. The total mass of dust in the disk with grain sizes between $a_{\min }$ and $a_{\max }$ is given by the $M_{\text {dust }}$ parameter. Each of the above parameters can be defined independently for the inner and outer disk components, which are separated by a wide gap.

The main goal of this Letter is to investigate the constraints the Herschel data provide on the outer disk properties of T Cha. As the Herschel data give no new information on the inner regions, we adopt the inner disk parameters from O11, which are listed in Table 2, as fixed values. The stellar parameters for $\mathrm{T}$ Cha are also taken from $\mathrm{O} 11$ and are listed in Table 2 as well. We also fix the minimum grain size for the outer disk and its inclination. We are thus left with seven free model parameters, all of which are related to the outer disk: $R_{\text {in }}, R_{\text {out }}, \alpha, \beta, a_{\max }, M_{\text {dust }}$, and $H_{50}$ (the scale height at $50 \mathrm{AU}$ ). Motivated by the lack of $10 \mu \mathrm{m}$ silicate emission in the Spitzer-IRS spectra of T Cha, O11 adopted for the inner disk a mixture of astro silicates (Draine $\&$ Lee 1984) and amorphous carbon (Zubko et al. 1996) with a mass ratio of 4:1 between silicate and carbon. For simplicity, we adopt the same silicate and carbon mix for the dust composition of the outer disk, but remind the reader that disk parameters derived from SED fitting are strongly dependent on the choice of dust properties. Although discussing the dependence of each parameter on dust composition is beyond the scope of this Letter, we have verified that our main conclusions remain unchanged if pure astro silicate grains are adopted for the outer disk.

\subsection{Model Grid and Best-fit Models}

In order to constrain the parameter space consistent with the observed $\mathrm{T}$ Cha SED, we ran a grid of models varying the seven free parameters in our model setup. The values sampled by the grid are listed in Table 3 . The parameters $\beta$ and $H_{50}$ are sampled linearly, while $R_{\text {in }}$ and $R_{\text {out }}, \alpha, a_{\max }$, and $M_{\text {dust }}$ are sampled logarithmically. The number of values per parameter range from 3 to 10 and the total number of models amount to 126,000. The parameters of the best-fit model (i.e., with the minimum $\chi^{2}$ ) are also listed in Table 3 . The global best-fit model so derived for the outer disk of $\mathrm{T}$ Cha is a narrow ring with an inner radius of $\sim 18 \mathrm{AU}$ and a width of only $\sim 2$ AU. This narrow ring remains optically thick even at (sub)millimeter wavelengths, which explains the very large mass that is allowed by the model. Its SED is shown as a solid black line in Figure 1.
Since SED modeling is known to be highly degenerate, the best-fit model is unlikely to be a unique solution. We therefore adopt the Bayesian method (Press et al. 1992; Pinte et al. 2008) to calculate the probability of different values for each parameter given the available data. We assume that we have no prior knowledge on the model parameter, which implies that the relative probability of a given model is proportional to $\exp \left[-\chi^{2} / 2\right]$. All probabilities are normalized so the sum of all the probabilities of the models in the grid is equal to 1. The top two panels in Figure 2 show the probability that each parameter takes a given value from the grid, calculated by summing over the probabilities of all the other parameters. Clearly, the $M_{\text {dust }}, a_{\max }$, and $R_{\text {in }}$ parameters are much better constrained than the others. The lower panels in Figure 2 show the joint probabilities of the $R_{\text {in }}, R_{\text {out }}$, and $\alpha$ parameters. Models with $R_{\text {in }} \sim 18 \mathrm{AU}$ and slightly larger $R_{\text {out }}$ values are clearly favored to best reproduce our observations. There are a large number of narrow ring models that can reproduce the observed T Cha SED relatively well. The best-fit model is an instance of a large family of such models. This can be understood considering that the SED of a narrow ring is little sensitive to parameters such as $\alpha, \beta$, or even $M_{\text {dust }}$ and $a_{\text {max }}$. Wider rings are also allowed, but they require fine-tuning of other parameters (see, for example, the red dashed line in Figure 1 and the $\alpha=-1$ model in Table 3) and thus appear as less likely models in the joint probability plots. Large disks $\left(R_{\text {out }} \gtrsim 150 \mathrm{AU}\right)$ with shallow surface density profiles $(\alpha \gtrsim$ $-1)$ result in a poor fit to the SED. This can be seen in Figure 2 in the dark region in the upper right corner of the $\alpha$ versus $R_{\text {out }}$ plot (lower row) and in the red histogram in the $\alpha$ probability distribution (middle row). We find that all such models result in far-IR Herschel colors that are much redder than observed (see bottom panel in Figure 2). In other words, all disk models with a shallow surface density profile $(\alpha \gtrsim-1)$ and a large outer radius (e.g., $\gtrsim 150-300 \mathrm{AU}$ ) either underpredict the observed 30 and $70 \mu \mathrm{m}$ fluxes and/or overpredict the 250 and $350 \mu \mathrm{m}$ fluxes. This is not surprising considering that a narrow ring around $\mathrm{T}$ Cha (like the best-fit model) would be "missing" most of the cool material emitting at 250 and 350 $\mu \mathrm{m}$ expected for a large disk with a relatively shallow surface density profile. However, a narrow ring is not the only way to reproduce the far-IR Herschel colors. We also find that the outer disk can be arbitrarily large (e.g., $R_{\text {out }}=300 \mathrm{AU}$ ) as long as the surface density profile is sufficiently steep (e.g., $\alpha=-3$ ) to quickly reduce the amount of dust at large radii. See, for example, the alternative $\alpha=-3$ model in Table 3, corresponding to the light blue dot-dashed line in Figure 1. This alternative model falls outside of our parameter grid, but illustrates well the strong degeneracy between the outer radius and the surface density profile of the disk. We note that while the $\alpha=-1$ and $\alpha=-3$ models have very different $R_{\text {out }}$ values, their $M_{\text {dust }}, R_{\text {in }}, a_{\max }, \beta$, and $H_{50}$ parameters are much more similar.

Independently of the probability space they occupy in Figure 2, the three models discussed above and listed in Table 3 are fully consistent with all the available data and, individually, can be considered to be equally good descriptions of the T Cha disk. One feature that our models cannot reproduce well is the pronounced V-shape of the Spitzer-IRS spectra around $15 \mu \mathrm{m}$. The models also underestimate the 2.2 and $3.6 \mu \mathrm{m}$ excesses from the inner disk, whose parameters are heavily driven by near-IR interferometry observations. Near-IR variability makes it difficult to simultaneously fit multi-epoch observations in this 

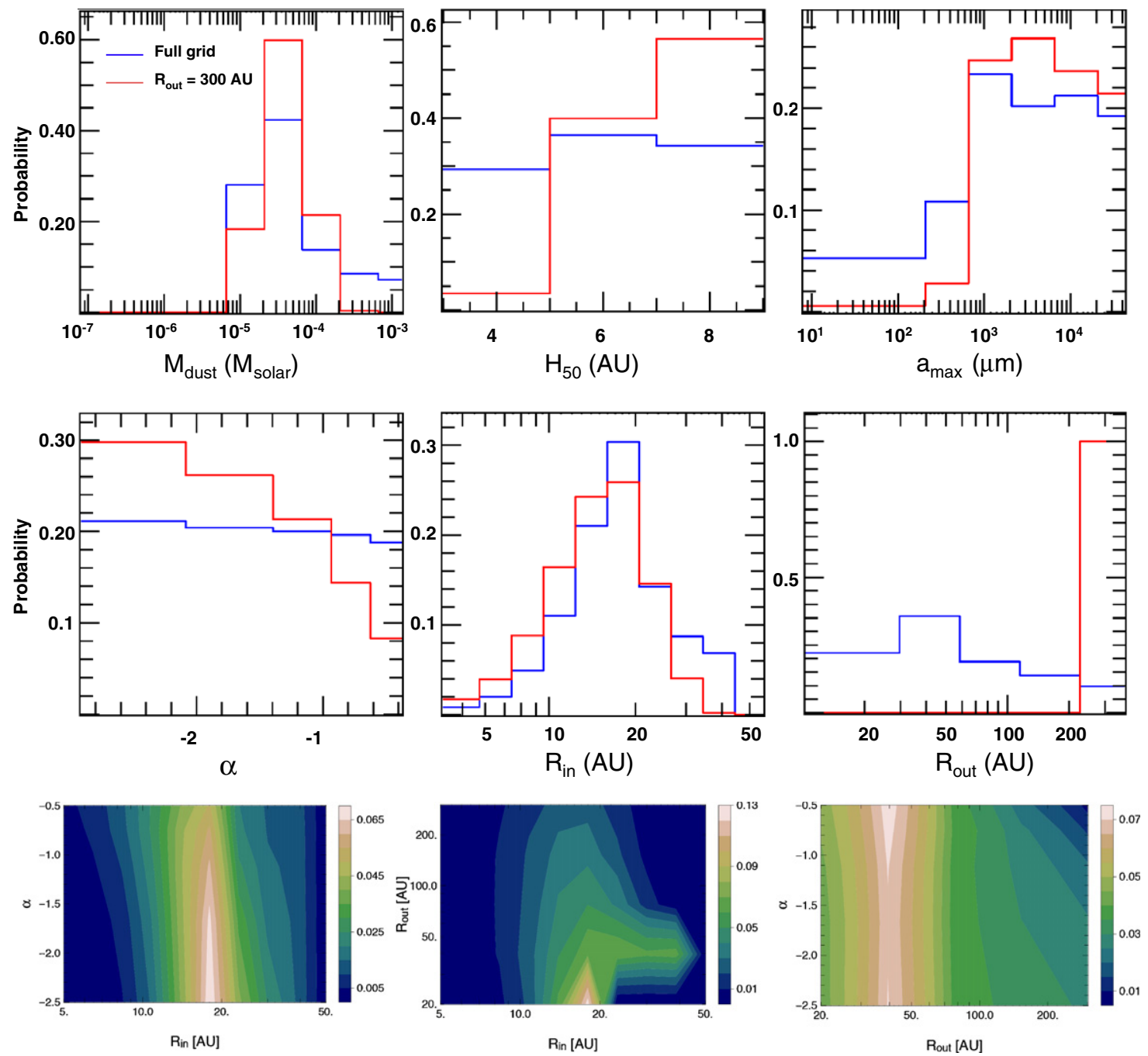

Figure 2. Top rows: Bayesian probabilities for six of the seven free parameters in our model for the entire grid (blue histograms) and the subset of models with $R_{\text {out }}=$ $300 \mathrm{AU}$ (red histograms). Due to space limitations, the $\beta$ parameter is not included, but it presents a rather flat probability distribution. Models with large $R_{\text {out }}$ clearly favor steep surface density profiles and larger $H_{50}$ values. Bottom row: two-dimensional probability maps of the full grid. The highest probability regions are indicated in bright colors. Many models with $R_{\text {in }} \sim 18$ AU and a steep surface density profile ( $\alpha \lesssim-1.5$ ) fit the data relatively well (left column). Similarly, a large number of narrow rings $\left(R_{\text {in }} \sim 18 \mathrm{AU}\right.$ and $R_{\text {in }} \lesssim 50 \mathrm{AU}$ ) can reproduce the observed SED (middle column). Models with $R_{\text {out }} \sim 40$ AU are most favored by the data. Disks with shallow surface density profiles $(\alpha \gtrsim-1.0)$ and large outer radius $\left(R_{\text {out }} \sim 150-300 \mathrm{AU}\right)$ result in poor fits to the SED (right column).

(A color version of this figure is available in the online journal.)

Table 3

Model Grid and Fitted Outer Disk Parameters

\begin{tabular}{llccc}
\hline \hline Parameter & \multicolumn{1}{c}{ Values Sampled by Grid } & Best-fit Model & $\alpha=-1$ Model $^{\mathrm{a}}$ & $\alpha=-3 \mathrm{Model}^{\mathrm{a}}$ \\
\hline Surface density profile $(\alpha)$ & $-2.5,-1.75,-1.1,0.75,0.5$ & -0.75 & -1 & -3 \\
$R_{\text {in }}(\mathrm{AU})$ & $4,5.5,7.5,11,15,18,25,30,40,50$ & 18 & 10.5 & 15 \\
$R_{\text {out }}(\mathrm{AU})$ & $20,40,80,150,300$ & 20 & 30 & 300 \\
$M_{\text {dust }}\left(M_{\odot}\right)$ & $(10,3,1,0.3,0.1,0.01,0.001) \times 10^{-4}$ & $1.0 \times 10^{-3}$ & $2.0 \times 10^{-5}$ & $1.8 \times 10^{-5}$ \\
Flaring index $(\beta)$ & $1.00,1.07,1.15$ & 1.15 & 5 & 1.1 \\
$H_{50}(H$ at 50 AU) (AU) & $4,6,8$ & 3 & 3 & 6 \\
$a_{\max }(\mathrm{mm})$ & $0.01,0.03,0.1,0.3,1,3,10,30$ & 3 & 3 \\
\hline
\end{tabular}

Note. ${ }^{\text {a }}$ The best-fit models fixing the value of $\alpha$ to -1 or -3 and fine-tuning the other parameters by hand guided by the results from the grid.

spectral region (see O11 for a detailed description of the inner disk modeling). Because the inner disk is optically thick, the exact geometry of the inner disk might affect some of the outer disk parameters like $H_{50}$ and $R_{\text {in }}$ (Mulders et al. 2010), but is unlikely to play any role on the values of $\alpha$ and $R_{\text {out }}$, the key parameters in this study.

\section{DISCUSSION}

The results from the previous section show that T Cha SED can be modeled with multiple combinations of parameter values. The most likely models can be divided into two broad categories: narrow rings and wider disks with steep surface density profiles. 
Both families of models suggest a highly unusual outer disk and have important but different implications for the nature of $\mathrm{T}$ Cha. A narrow ring would strongly suggest that the outer disk has been truncated by the dynamical interaction of a companion, as is known to be the case in the HD 98800B and Hen 3-600A multiple systems, which have outer radii of only 10 and $20 \mathrm{AU}$, respectively (Andrews et al. 2010). Very deep imaging of T Cha (Chauvin et al. 2010; Vicente et al. 2011) rule out any stellar companion that could be responsible for the truncation of the outer disk, unless its projected separation is currently less than 0 '.1 (10 AU). Furthermore, recent aperture masking observations by Huélamo et al. (2011) only report a very lowmass companion candidate (either a young planet or a brown dwarf) with a projected separation of $6.2 \pm 0.7$ AU. Since this object is the most likely cause of the inner cavity $\left(R_{\text {in }} \sim\right.$ 10-20 AU based on the SED modeling), its physical separation must be $<R_{\text {in }}$. Any object truncating the outer disk thus must be substellar or planetary in nature.

We speculate that the recently reported companion to $\mathrm{T}$ Cha could potentially also provide the explanation for the models with $\alpha \lesssim-2$. These unusual $\alpha$ values would imply that the disk material is highly concentrated toward the inner edge of the outer disk (i.e., that material is accumulating at the outer edge of the gap, at $\sim 15 \mathrm{AU})$. As a reference, $\alpha=-1.5$ in the canonical Minimum Mass Solar Nebula (Weidenschilling 1977). T Cha's outer disk is relatively massive but shows little or no accretion onto the star (Alcalá et al. 1993; Schisano et al. 2009). The transport of angular momentum and material across the outermost part of the disk should not be affected by the presence of the low-mass object, but when circumstellar material reaches the outer edge of the gap, it can be accreted by the low-mass object, channeled toward the inner disk, or accumulated at the gap's edge (Lubow \& D'Angelo 2006). The dynamical interaction between a gas-rich disk and a planetary mass object is a complex hydrodynamic problem that depends on the mass and location of the planet as well as on the properties of the disk (viscosity, disk scale height, etc.), but an $\alpha$ value of $\lesssim-2$ would suggest that it could have an important effect on the surface density of the outer disk. Recent threedimensional hydrodynamic models of planet-disk interactions show the accumulation of millimeter-sized grains in the pressure maxima of the gas caused by the spiral density waves triggered by the planet (Fouchet et al. 2010). This implies that the density enhancement at the edge of the gap of the large grains traced by Herschel could be even larger than it is for the gas. However, given the degeneracy between $\alpha$ and $R_{\text {out }}$, the dynamical effect of the companion on the disk cannot be tested without spatially resolved observations. Our models show that, given the SED data alone and without knowing $R_{\text {out }}$, the value of $\alpha$ for the dust disk could be anywhere between -0.75 and -3 .

\section{CONCLUSIONS}

New Herschel observations of T Cha reveal a steep spectral slope between 70 and $350 \mu \mathrm{m}$ (i.e., very blue far-IR Herschel colors compared to a "normal disk"). The steeply decreasing far-IR SED is not due to a lack of large grains. In fact, the (sub)millimeter wavelength colors are rather red and grains larger than $\sim 1 \mathrm{~mm}$ are needed to simultaneously fit the Herschel and 1.3 and $3.3 \mathrm{~mm}$ fluxes. Instead, we find basically two ways to reproduce the observed far-IR Herschel colors: making the outer disk very small (by reducing the value of $R_{\text {out }}$ ) or very tenuous (by reducing the value of the surface density profile, $\alpha$ ). Both types of models contain little or no dust beyond $\sim 40$ AU, but each family has a different astrophysical implication: either $\mathrm{T}$ Cha has a small outwardly truncated disk or material is accumulating at the inner edge of the gap. Resolved images are mandatory to break the degeneracy between $\alpha$ and the inner and outer disk radii. The Atacama Large Millimeter/submillimeter Array will have both the resolution and sensitivity needed to directly measure the width of the disk as well as its surface density profile and distinguish between the two interpretations. The former result would encourage even deeper searches for a wide-separation low-mass companion (most likely a Jovian planet). The latter scenario would have important implications for the dynamical interaction between the T Cha disk and the recently reported companion as well as on any subsequent planet formation in its highly peculiar outer disk.

Support for this work, part of the DIGIT Herschel Open Time Key Program, was provided by NASA through an award issued by JPL/Caltech. L.A.C. acknowledges support from NASA through the Sagan Fellowship Program. J.O. acknowledges a grant from the Alexander von Humboldt Foundation. C.P., J.C.A., and F.M. acknowledge funding from the European Commission's 7th Framework Program and the Agence Nationale pour la Recherche of France (projects FP7: PERG06-GA-2009256513, ANR-07-BLAN-0221, and ANR-2010-JCJC-0504-01) and Programme National de Physique Stellaire (PNPS) of CNRS/INSU, France.

\section{REFERENCES}

Alcalá, J. M., Covino, E., Franchini, M., et al. 1993, A\&A, 272, 225 Andrews, S. M., Czekala, I., Wilner, D. J., et al. 2010, ApJ, 710, 462 Brown, J. M., Blake, G. A., Dullemond, C. P., et al. 2007, ApJ, 664, L107 Chauvin, G., Lagrange, A.-M., Bonavita, M., et al. 2010, A\&A, 509, A52 Draine, B. T., \& Lee, H. M. 1984, ApJ, 285, 89

Espaillat, C., D’Alessio, P., Hernández, J., et al. 2010, ApJ, 717, 441

Evans, N. J., II., Harvey, P. M., Dunham, M. M., et al. 2007, Final Delivery of Data from the c2d Legacy Project: IRAC and MIPS (Pasadena, CA: SSC)

Fouchet, L., Gonzalez, J.-F., \& Maddison, S. T. 2010, A\&A, 518, A16

Griffin, M. J., Abergel, A., Abreu, A., et al. 2010, A\&A, 518, L3

Huélamo, N., Lacour, S., Tuthill, P., et al. 2011, A\&A, 528, L7

Lommen, D., Wright, C. M., Maddison, S. T., et al. 2007, A\&A, 462, 211

Lubow, S. H., \& D’Angelo, G. 2006, ApJ, 641, 526

Merín, B., Brown, J. M., Oliveira, I., et al. 2010, ApJ, 718, 1200

Mulders, G. D., Dominik, C., \& Min, M. 2010, A\&A, 512, A11

Najita, J. R., Strom, S. E., \& Muzerolle, J. 2007, MNRAS, 378, 369

Olofsson, J., Benisty, M., Augereau, J.-C., et al. 2011, A\&A, 528, L6

Ott, S. 2010, in ASP Conf. Ser. 434, Astronomical Data Analysis Software and Systems XIX, ed. Y. Mizumoto, K. Morita, \& M. Ohishi (San Francisco, CA: ASP), 139

Pinte, C., Harries, T. J., Min, M., et al. 2009, A\&A, 498, 967

Pinte, C., Ménard, F., Duchêne, G., \& Bastien, P. 2006, A\&A, 459, 797

Pinte, C., Padgett, D. L., Ménard, F., et al. 2008, A\&A, 489, 633 Poglitsch, A., Waelkens, C., Geis, N., et al. 2010, A\&A, 518, L2

Press, W. H., Teukolsky, S. A., Vetterling, W. T., \& Flannery, B. P. 1992, Numerical Recipes in FORTRAN (2nd ed.; Cambridge: Cambridge Univ. Press)

Schisano, E., Covino, E., Alcalá, J. M., et al. 2009, A\&A, 501, 1013

Scholler, M. 2007, New Astron. Rev., 51, 628

Skrutskie, M. F., Cutri, R. M., Stiening, R., et al. 2006, AJ, 131, 1163

Strom, K. M., Strom, S. E., Edwards, S., Cabrit, S., \& Skrutskie, M. F. 1989, AJ, 97, 1451

Vicente, S., Merín, B., Hartung, M., et al. 2011, A\&A, 533, A135

Weidenschilling, S. J. 1977, Ap\&SS, 51, 153

Williams, J. P., \& Cieza, L. A. 2011, ARA\&A, 49, 67

Zubko, V. G., Mennella, V., Colangeli, L., \& Bussoletti, E. 1996, MNRAS, 282, 1321 